\title{
ON THE LIE STRUCTURE OF LOCALLY MATRIX ALGEBRAS
}

\section{BEZUSHCHAK O.}

Let $A$ be a unital locally matrix algebra over a field $\mathbb{F}$ of characteristic different from 2 . We find a necessary and sufficient condition for the Lie algebra $A / \mathbb{F} \cdot 1$ to be simple and for the Lie algebra of derivations $\operatorname{Der}(A)$ to be topologically simple. The condition depends on the Steinitz number of $A$ only.

Key words and phrases: locally matrix algebra, derivation.

Taras Shevchenko National University of Kyiv, 64/13 Volodymyrska str., 01601, Kyiv, Ukraine

E-mail: bezuscheuniv.kiev.ua

\section{INTRODUCTION}

Let $F$ be a ground field of characteristic different from 2 and let $\mathbb{N}$ be the set of all positive integers. Recall that an associative $F$-algebra $A$ is called a locally matrix algebra (see $[9,10])$ if for an arbitrary finite subset of $A$ there exists a subalgebra $B \subset A$ containing this subset and such that $B \cong M_{n}(F)$ for some $n \in \mathbb{N}$. We call a locally matrix algebra unital if it contains unit 1 .

Let $\mathbb{P}$ be the set of all primes. An infinite formal product of the form

$$
s=\prod_{p \in \mathbb{P}} p^{r_{p}}, \quad \text { where } \quad r_{p} \in \mathbb{N} \cup\{0, \infty\} \quad \text { for all } \quad p \in \mathbb{P},
$$

is called Steinitz number; see [12]. Denote by symbol SN the set of all Steinitz numbers. Let

$$
s_{1}=\prod_{p \in \mathbb{P}} p^{r_{p}}, s_{2}=\prod_{p \in \mathbb{P}} p^{k_{p}} \in \mathbb{S N}
$$

Then

$$
s_{1} \cdot s_{2}=\prod_{p \in \mathbb{P}} p^{r_{p}+k_{p}}, \quad \text { where } k_{p} \in \mathbb{N} \cup\{0, \infty\},
$$

and $t+\infty=\infty+t=\infty+\infty=\infty$ for all $t \in \mathbb{N}$.

Let $A$ be a countable-dimensional unital locally matrix algebra. In [5], J.G. Glimm defined the Steinitz number st $(A)$ of the algebra $A$ and proved that the algebra $A$ is uniquely determined by $\mathbf{s t}(A)$.

In [2], we extended Glimm's definition to unital locally matrix algebras of arbitrary dimensions. For a unital locally matrix algebra $A$ denote by $D(A)$ the set of all numbers $n \in \mathbb{N}$ such that there exists a subalgebra $A^{\prime} \subseteq A, 1 \in A^{\prime}$, and $A^{\prime} \cong M_{n}(F)$. The Steinitz number $\operatorname{st}(A)$ of the algebra $A$ is the least common multiple of the set $D(A)$. It turned out that a unital locally matrix algebra $A$ of dimension $>\aleph_{0}$ is no longer determined by its Steinitz number st $(A)$; see $[2,3]$. 
An associative algebra $A$ gives rise to the Lie algebra

$$
A^{(-)}=(A,[a, b]=a b-b a) .
$$

Along with the Lie algebra $A^{(-)}$we will consider its square $[A, A]$. Let $Z(A)$ denote the center of the associative algebra $A$. In [6], I.N. Herstein showed that if $A$ is a simple associative algebra then the Lie algebra

$$
[A, A] / Z(A) \cap[A, A]
$$

is simple. Since a locally matrix algebra $A$ is simple, it follows that the Lie algebra (2) is simple.

Let $M_{n}(\mathbb{F})$ be a matrix algebra, $\mathfrak{g l}(n)=\left(M_{n}(\mathbb{F})\right)^{(-)}, Z\left(M_{n}(\mathbb{F})\right)=\mathbb{F} \cdot 1$. The Lie algebra

$$
\mathfrak{p g l}(n)=\mathfrak{g l}(n) / \mathbb{F} \cdot 1
$$

is simple unless $p=$ char $\mathbb{F}>0$ and $p$ divides $n$; see [11]. We will show that for an infinitedimensional unital locally matrix algebra $A$ simplicity of the Lie algebra

$$
A^{(-)} / \mathbb{F} \cdot 1
$$

depends only on the Steinitz number st $(A)$.

For a Steinitz number (1) denote $v_{p}(s)=r_{p}$.

Theorem 1. The Lie algebra (3) is simple if and only if

$$
\operatorname{char} \mathbb{F}=0
$$

or

$$
\text { char } \mathbb{F}=p>0 \quad \text { and } \quad v_{p}(\operatorname{st}(A))=0 \quad \text { or } \quad \infty .
$$

Recall that a linear transformation $d: A \rightarrow A$ of an algebra $A$ is called a derivation if

$$
d(a b)=d(a) \cdot b+a \cdot d(b)
$$

for arbitrary elements $a, b \in A$. The vector space $\operatorname{Der}(A)$ of all derivations of an algebra $A$ is a Lie algebra with respect to commutation; see [7]. If $A$ is an associative algebra then for an arbitrary element $a \in A$ the operator

$$
\operatorname{ad}(a): A \rightarrow A, \quad x \mapsto[a, x],
$$

is an inner derivation. The subspace $\operatorname{Inder}(A)=\{\operatorname{ad}(a) \mid a \in A\}$ of all inner derivations is an ideal of the Lie algebra $\operatorname{Der}(A)$.

Let $X$ be an arbitrary set. The set $\operatorname{Map}(X, X)$ of all mappings $X \rightarrow X$ is equipped with Tykhonoff topology; see [13]. The subspace of all derivations $\operatorname{Der}(A)$ of an algebra $A$ is closed in $\operatorname{Map}(A, A)$ in Tykhonoff topology. It makes the Lie algebra $\operatorname{Der}(A)$ a topological algebra.

Theorem 2. Let $A$ be a unital locally matrix algebra. Then

(1) the Lie algebra $[\operatorname{Der}(A), \operatorname{Der}(A)]$ is topologically simple;

(2) the Lie algebra $\operatorname{Der}(A)$ is topologically simple if and only if char $\mathbb{F}=0$ or char $\mathbb{F}=p>0$ and $v_{p}(\mathbf{s t}(A))=0$ or $\infty$. 


\section{ProOf OF THE THEOREM 1}

Proof. Let $A$ be a unital locally matrix $\mathbb{F}$-algebra. We will show that

$$
\begin{aligned}
& A=[A, A]+\mathbb{F} \cdot 1 \quad \text { if and only if } \operatorname{char} \mathbb{F}=0 \\
& \text { or } \quad \operatorname{char} \mathbb{F}=p>0 \text { and } \quad v_{p}(\operatorname{st}(A))=0 \text { or } \infty .
\end{aligned}
$$

Consider a matrix algebra $M_{n}(\mathbb{F})$. Then $\left[M_{n}(\mathbb{F}), M_{n}(\mathbb{F})\right]=\left\{a \in M_{n}(\mathbb{F}) \mid \operatorname{tr}(a)=0\right\}=$ $\mathfrak{s l}(n)$. If char $\mathbb{F}=0$ or char $\mathbb{F}=p>0$ and $p$ does not divide $n$ then $\operatorname{tr}(1)=n \neq 0$, and therefore $a=\left(a-\frac{1}{n} \operatorname{tr}(a) \cdot 1\right)+\frac{1}{n} \operatorname{tr}(a) \cdot 1 \in \operatorname{sl}(n)+\mathbb{F} \cdot 1$ for an arbitrary element $a \in A$. It implies that

$$
M_{n}(\mathbb{F})=\left[M_{n}(\mathbb{F}), M_{n}(\mathbb{F})\right]+\mathbb{F} \cdot 1
$$

If $p$ divides $n$ then $\operatorname{tr}(1)=0$, hence

$$
\left[M_{n}(\mathbb{F}), M_{n}(\mathbb{F})\right]+\mathbb{F} \cdot 1=\mathfrak{s l}(n) \neq M_{n}(\mathbb{F}) .
$$

If char $\mathbb{F}=0$ or char $\mathbb{F}=p>0$ and $p$ does not divide st $(A)$ then for an arbitrary matrix subalgebra $1 \in A_{1} \subset A, A_{1} \cong M_{n}(\mathbb{F})$, the characteristic $p$ does not divide $n$. Hence

$$
A_{1}=\left[A_{1}, A_{1}\right]+\mathbb{F} \cdot 1 .
$$

So, $A=[A, A]+\mathbb{F} \cdot 1$.

Suppose now that $p^{\infty}$ divides $\mathbf{s t}(A)$. Consider a matrix subalgebra

$$
1 \in A_{1} \subset A, \quad A_{1} \cong M_{n}(\mathbb{F}) .
$$

The number $n$ divides $\mathbf{s t}(A)$. Since $p^{\infty}$ divides $\mathbf{s t}(A)$ it follows that $p n$ also divides $\mathbf{s t}(A)$. Hence, there exists a subalgebra $A_{2} \subset A$ such that

$$
A_{1} \subset A_{2}, \quad A_{2} \cong M_{m}(\mathbb{F}) \text { and } p \text { divides } m / n \text {. }
$$

Let $C$ be the centralizer of the subalgebra $A_{1}$ in $A_{2}$. We have

$$
A_{2}=A_{1} \otimes_{\mathbb{F}} C, \quad C \cong M_{m / n}(\mathbb{F})
$$

(see $[4,8])$. For arbitrary elements $a \in A_{1}, b \in C$ we have

$$
\operatorname{tr}_{A_{2}}(a b)=\operatorname{tr}_{A_{1}}(a) \cdot \operatorname{tr}_{C}(b)
$$

where $\operatorname{tr}_{A_{2}}, \operatorname{tr}_{A_{1}}, \operatorname{tr}_{C}$ are traces in the subalgebras $A_{2}, A_{1}, C$, respectively. We have

$$
\operatorname{tr}_{C}(1)=\frac{m}{n}=0
$$

Hence,

$$
\operatorname{tr}_{A_{2}}\left(A_{1} \otimes 1\right)=\operatorname{tr}_{A_{1}}\left(A_{1}\right) \cdot \operatorname{tr}_{C}(1)=\{0\},
$$

and therefore $A_{1} \subseteq\left[A_{2}, A_{2}\right]$. We showed that if $p^{\infty}$ divides st $(A)$ then $A=[A, A]$.

Suppose now that $v_{p}(\mathbf{s t}(A))=k, 1 \leq k<\infty$. Consider a subalgebra

$$
1 \in A_{1} \subset A, \quad A_{1} \cong M_{p^{k}}(\mathbb{F}) .
$$


Choose an element $a \in A_{1}$ such that $\operatorname{tr}_{A_{1}}(a) \neq 0$. We claim that

$$
a \notin[A, A]+\mathbb{F} \cdot 1 \text {. }
$$

Indeed, if the element $a$ lies in the right hand side then there exists a subalgebra $A_{2} \subset A$ such that

$$
A_{1} \subset A_{2}, \quad A_{2} \cong M_{n}(\mathbb{F}) \text { and } a \in\left[A_{2}, A_{2}\right]+\mathbb{F} \cdot 1 .
$$

Since $p$ divides $n$ it follows that $\operatorname{tr}_{A_{2}}(1)=0$, hence $\operatorname{tr}_{A_{2}}(a)=0$.

As above, let $C$ be the centralizer of the subalgebra $A_{1}$ in $A_{2}$, so that $A_{2}=A_{1} \otimes_{\mathbb{F}} C$. The algebra $C$ is isomorphic to the matrix algebra $M_{m}(\mathbb{F})$, where $m=n / p^{k}$. The number $m$ is coprime with $p$, hence $\operatorname{tr}_{C}(1)=m \neq 0$. Now,

$$
\operatorname{tr}_{A_{2}}(a)=\operatorname{tr}_{A_{2}}(a \otimes 1)=\operatorname{tr}_{A_{1}}(a) \cdot \operatorname{tr}_{C}(1) \neq 0 .
$$

This contradiction completes the proof of the claim (4).

If $A=[A, A]+\mathbb{F} \cdot 1$ then

$$
A^{(-)} / \mathbb{F} \cdot 1 \cong[A, A] /[A, A] \cap \mathbb{F} \cdot 1 .
$$

In this case, the Lie algebra $A^{(-)} / \mathbb{F} \cdot 1$ is simple by I.N. Herstein's Theorem (see [6]). If $A=[A, A]+\mathbb{F} \cdot 1$ is a proper subspace of $A$ then

$$
[A, A]+\mathbb{F} \cdot 1 / \mathbb{F} \cdot 1
$$

is a proper ideal in the Lie algebra $A^{(-)} / \mathbb{F} \cdot 1$. This completes the proof of Theorem 1.

Consider the homomorphism

$$
\varphi: A^{(-)} \rightarrow \operatorname{Inder}(A), \quad \varphi(a)=\operatorname{ad}(a), \quad a \in A .
$$

Since $\operatorname{Ker} \varphi=Z(A)=\mathbb{F} \cdot 1$ it follows that $\operatorname{Inder}(A) \cong A^{(-)} / \mathbb{F} \cdot 1$.

Corollary 1. (1) The Lie algebra $[\operatorname{Inder}(A), \operatorname{Inder}(A)]$ is simple.

(2) The Lie algebra Inder $(A)$ is simple if and only if char $\mathbb{F}=0$ or char $\mathbb{F}=p>0$ and $v_{p}(\mathbf{s t}(A))=0$ or $\infty$.

Proof. The Lie algebra

$$
[\operatorname{Inder}(A), \operatorname{Inder}(A)] \cong[A, A] /[A, A] \cap \mathbb{F} \cdot 1
$$

is simple by I.N. Herstein's Theorem (see [6]). The part (2) immediately follows from Theorem 1.

\section{PROOF OF THE THEOREM 2}

Lemma 1. Let $A$ be an infinite-dimensional locally matrix algebra. Let $d \in \operatorname{Der}(A)$ and suppose that $d([A, A])$ lies in the center of the algebra $A$. Then $d=0$. 
Proof. Let $Z$ be the center of $A$. If $A$ is not unital then $Z=\{0\}$. If $A$ is unital then $Z=\mathbb{F} \cdot 1$. Consider a subalgebra $A_{1} \subset A$ such that $A_{1} \cong M_{n}(\mathbb{F})$ for some $n \geq 4$, and let $\varphi: M_{n}(\mathbb{F}) \rightarrow A_{1}$ be an isomorphism. An arbitrary matrix unit $e_{i j}, 1 \leq i \neq j \leq n$, lies in $\left[M_{n}(\mathbb{F}), M_{n}(\mathbb{F})\right]$. Choose distinct indices $1 \leq i, j, s, t \leq n$. Then $e_{i j}=\left[e_{i s}, e_{s j}\right]$. Hence,

$$
d\left(\varphi\left(e_{i j}\right)\right) \in Z \varphi\left(e_{i s}\right)+Z \varphi\left(e_{s j}\right) .
$$

On the other hand, $e_{i j}=\left[e_{i t}, e_{t j}\right]$, which implies $d\left(\varphi\left(e_{i j}\right)\right) \in Z \varphi\left(e_{i t}\right)+Z \varphi\left(e_{t j}\right)$. Hence, $d\left(\varphi\left(e_{i j}\right)\right)=0$. The algebra $M_{n}(\mathbb{F})$ is generated by matrix units $e_{i j}, 1 \leq i \neq j \leq n$. So, $d\left(\varphi\left(M_{n}(\mathbb{F})\right)\right)=\{0\}$, and therefore $d(A)=\{0\}$. This completes the proof of the Lemma.

In [1], we showed that for an arbitrary locally matrix algebra $A$ the ideal $\operatorname{Inder}(A)$ is dense in the Lie algebra $\operatorname{Der}(A)$ in the Tykhonoff topology.

Proof of Theorem 2. (1) Let $I$ be a nonzero closed ideal of the Lie algebra $[\operatorname{Der}(A), \operatorname{Der}(A)]$. Choose a nonzero element $d \in I$. For an arbitrary element $a \in[A, A]$ we have

$$
[d, \operatorname{ad}(a)]=\operatorname{ad}(d(a)) \in[\operatorname{Inder}(A), \operatorname{Inder}(A)] .
$$

By Lemma 1, we can choose an element $a \in[A, A]$ so that $d(a) \neq 0$. Hence,

$$
I \cap[\operatorname{Inder}(A), \operatorname{Inder}(A)] \neq\{0\} .
$$

Since the Lie algebra $[\operatorname{Inder}(A)$, Inder $(A)]$ is simple it follows that

$$
[\operatorname{Inder}(A), \operatorname{Inder}(A)] \subseteq I .
$$

We have mentioned above that $\operatorname{Inder}(A)$ is dense in the Lie algebra $\operatorname{Der}(A)$ in the Tykhonoff topology; see [1]. Hence, $[\operatorname{Inder}(A), \operatorname{Inder}(A)]$ is dense in the Lie algebra $[\operatorname{Der}(A), \operatorname{Der}(A)]$. Since the ideal $I$ is closed we conclude that $I=[\operatorname{Inder}(A), \operatorname{Inder}(A)]$.

(2) Let $I$ be a nonzero closed ideal of the Lie algebra $\operatorname{Der}(A)$. Choose a nonzero derivation $d \in I$. By Lemma 1 , there exists an element $a \in A$ such that $d(a)$ does not lie in $\mathbb{F} \cdot 1$, hence

$$
0 \neq \operatorname{ad}(d(a))=[d, \operatorname{ad}(a)] \in I \cap \operatorname{Inder}(A) .
$$

Suppose that char $\mathbb{F}=0$ or char $\mathbb{F}=p>0$ and $v_{p}(\mathbf{s t}(A))=0$ or $\infty$. Then the Lie algebra Inder $(A)$ is simple, and therefore $\operatorname{Inder}(A) \subseteq I$. Since $\operatorname{Inder}(A)$ is dense in $\operatorname{Der}(A)$ (see [1]) and the ideal $I$ is closed it follows that $I=\operatorname{Der}(A)$.

Now suppose that $v_{p}(\operatorname{st}(A))=k, 1 \leq k<\infty$. There exists a subalgebra $A_{1}$ in $A$ such that

$$
1 \in A_{1} \quad \text { and } \quad A_{1} \cong M_{p^{k}}(\mathbb{F})
$$

Choose an element $a \in A_{1}$ such that $\operatorname{tr}_{A_{1}}(a) \neq 0$. We will show that the inner derivation $\operatorname{ad}(a)$ does not lie in the closure

$$
\overline{[\operatorname{Der}(A), \operatorname{Der}(A)]}=\overline{[\operatorname{Inder}(A), \operatorname{Inder}(A)]},
$$

and therefore $\overline{[\operatorname{Inder}(A), \operatorname{Inder}(A)]}$ is a proper closed ideal in the Lie algebra $\operatorname{Der}(A)$. If $\operatorname{ad}(a)$ lies in the closure of $[\operatorname{Inder}(A), \operatorname{Inder}(A)]$ then, by the definition of the Tykhonoff topology, there exist elements $a_{i}, b_{i} \in A, 1 \leq i \leq n$, such that

$$
\left(\operatorname{ad}(a)-\sum_{i=1}^{n} \operatorname{ad}\left(\left[a_{i}, b_{i}\right]\right)\right)\left(A_{1}\right)=\{0\} .
$$


There exists a subalgebra $A_{2} \subset A$ such that

$$
A_{1} \subseteq A_{2}, \quad a, a_{i}, b_{i} \in A_{2}, \quad 1 \leq i \leq n, \quad \text { and } \quad A_{2} \cong M_{m}(\mathbb{F}) .
$$

As above, we consider the centralizer $C$ of the subalgebra $A_{1}$ in $A_{2}$ such that

$$
A_{2}=A_{1} \otimes_{\mathbb{F}} C, \quad C \cong M_{t}(\mathbb{F}) \text { and } \quad t=m / p^{k} \text { is not a multiple of } p .
$$

Consider the element

$$
b=\sum_{i=1}^{n}\left[a_{i}, b_{i}\right] \in A_{2} .
$$

The difference $a-b$ commutes with all elements from $A_{1}$, hence $a-b=c \in C$.

In the algebra $A_{2}$ we have

$$
\operatorname{tr}_{A_{2}}(c)=\operatorname{tr}_{A_{2}}(1 \otimes c)=\operatorname{tr}_{A_{1}}(1) \cdot \operatorname{tr}_{C}(c)=0 .
$$

Hence, $\operatorname{tr}_{A_{2}}(a)=\operatorname{tr}_{A_{2}}(b)+\operatorname{tr}_{A_{2}}(c)=0$.

On the other hand, $\operatorname{tr}_{A_{2}}(a)=\operatorname{tr}_{A_{2}}(a \otimes 1)=\operatorname{tr}_{A_{1}}(a) \cdot t \neq 0$. This contradiction completes the proof of Theorem 2 .

\section{REFERENCES}

[1] Bezushchak O. Derivations and automorphisms of locally matrix algebras. arXiv:2007.15716v1 [math.RA]

[2] Bezushchak O., Oliynyk B. Unital locally matrix algebras and Steinitz numbers. J. Algebra Appl. 2020, 19 (09), 2050180. doi:10.1142/S0219498820501807

[3] Bezushchak O., Oliynyk B. Primary decompositions of unital locally matrix algebras. Bull. Math. Sci. 2020, 10 (1), 2050006 (7 pages). doi:10.1142/S166436072050006X

[4] Drozd Yu.A., Kirichenko V.V. Finite Dimensional Algebras. Springer-Verlag, Berlin-Heidelberg-New York, 1994.

[5] Glimm J.G. On a certain class of operator algebras. Trans. Amer. Math. Soc. 1960, 95 (2), 318-340.

[6] Herstein I.N. On the Lie and Jordan rings of a simple associetive ring. Amer. J. Math. 1955, 77 (2), $279-285$.

[7] Jacobson N. Lie algebras. Dover Publications Inc., New Yourk, 1979.

[8] Jacobson N. Structure of rings. Colloquium Publications, 37, 1956.

[9] Köthe G. Schiefkörper unendlichen Ranges uber dem Zentrum. Math. Ann. 1931, 105, 15-39.

[10] Kurosh A. Direct decompositions of simple rings. Rec. Math. 1942, 11 (53) (3), 245-264.

[11] Seligman G.B. Modular Lie Algebras. Springer-Verlag, Berlin-Heidelberg, 1967.

[12] Steinitz E. Algebraische Theorie der Körper. J. Reine Angew. Math. 1910, 137, 167-309.

[13] Willard S. General Topology. Dover Publications, Mineola, New York, 2004.

Received 07.08.2020

Безущак О. Про лієву структуру локально матричних алгебр // Карпатські матем. публ. - 2020. — Т.12, №2. - С. 311-316.

Нехай $A-$ унітальна локально матрична алгебра над полем $\mathbb{F}$ характеристики відмінної від 2. Знайдено необхідну і достатню умову того, шоб алгебра $\Lambda$ i $A / \mathbb{F} \cdot 1$ була простою, а алгебра $\Lambda$ і диференціювань $\operatorname{Der}(A)$ - топологічно простою. Сформульована умова залежить лише від числа Стейніца алгебри $A$.

Ключові слова і фрази: локально матрична алгебра, диференціювання. 